(Aus der Universitätshautklinik Breslau [Dir.: Geh. Rat Prof. Dr. Jadassohn].)

\title{
Ein Fall von ulceröser Hauterkrankung aus der Gruppe des Ecthyma gangraenosum (mit Pyocyaneusbefund).
}

\author{
Von \\ Dr. Wilhelm Frei und Dr. Kurt Wiener, \\ Assistenten der Klinik. \\ Mit 1 Textabbildung. \\ (Eingegangen am 27. September 1920.)
}

Im Sommer des vorigen Jahres wurde in die hiesige Klinik eine Frau mit einer schweren uleerösen Hauterkrankung aufgenommen. Der Fall bot ein sehr außergewöhnliches Krankheitsbild und gab Anlaß zu verschiedenen bakteriologischen und serologischen Untersuchungen, besonders über das pathogene Verhalten des Bacillus pyocyaneus. Seine Veröffentlichung erschien uns deswegen angezeigt.

Aus der ansführlichen Krankengeschichte geben wir das Folgende wieder:

54 jährige Erau, seit 27 Jahren verbeiratet. 8 Geburten, 4 Kinder leben. Keine Anhaltspunkte für Lues, Tuberkulose oder andere wichtige Erkrankungen sowohl in der Familien- wie in der persônlichen Anamnese.

Im Juli 1918 entstanden zunächst auf der Brust und am Rücken ungefähr fünf kirschgroße eitrige Knoten, die nach 4 Wochen abheilten. Nov. 1918 ein Geschwür von Handtellergröße am linken Unterschenkel, das erst nach 4 Monaten zuheilte. Januar 1919 ein weiteres am linken Unterschenkel, später fünfmarkstückbis handtellərgroße am rechten Unterschenkel, an Banch, Brust, Rücken, die trotz ärztlicher Bahandlung schlecht verheilten. Es bestehen immer noch Gesehwüre an Brust und Räcken, die spontan starke Schmerzen verursachen. Seit Beginn der Erkrankung fühlt sich die Frau schwächer als sonst.

Befund: Mittelgroße Frau in mäBigem Ernährungszustand $(51,5 \mathrm{~kg})$, jedoch nicht kachektisch. Haut blaßß, etwas bräunlieh, schlaff. Gesioht blaß. Mtund- und Nasenschleimhaut o. B.

Ausgebildete und entstehende Efflorescenzen: über dem Sternum ein handtellergroßэs Gsschwür mit grüneitrigem Grunde und prominenten, bläulich glänzenden, unterminiorten, serpiginösen, mäßig infiltrierten Rändern. Über dem linken Sternoclaviculargelenk, an der rechten Mamma, an der linken Schulter und links von der Mitte der Wirbelsäule pfennig- bis talergroße ähnliche Geschwüre mit zum Teil məhr erhabanэm kraterartigem Rande von wechselnder Infiltration. Die linke Hälite der linken Mxmma ist von einem etwa $10 \times 20 \mathrm{~cm}$ großen Geschwür eingenommon, dessen Rïnder flach und unterminiert sind, und dessen Grund mit grünlichem, fest anhaftendem Eiter belegt ist. In dieser glatten Fläche finden sich wiederum kleine scharf ausgestanzte Geschwüre mit senkrechten Wänden und serös- 
eitrig bedecktem Grunde. In der Umgebung dieses Herdes vereinzelte erbsengroße, derb infiltrierte, blaurote Knötchen, die teilweise in der Mitte eine stecknadelkopfgroße Pustel zeigen. Einige kleine Herde am Bauch haben eine braunrote Kruste.

Narben: Auf der Brust mehrere langgestreckte unregelmäßig umarandete, teilweise mit streifenförmigen Ausläufern versehene, linsen- bis über handtellergroße weiße verschiebliche Narben. Besonders auffallend ist eine $10 \mathrm{~cm}$ lange ovale weiße Narbe oberhalb der rechten Mamma. Ähnliche, zum Teil in der Umgebung pigmentierte, Narben am Rücken, in der Gegend des linken Oberschenkelkopfes, am Bauch, am Kreuz und über der Analfurche. Einige zeigen einen etwas erhabenen Rand. Am rechten Unterschenkel innen dicht unterhalb der Patella ein kleinhandtellergroßer, kreisrunder, roter, flacher, leicht schuppender, infiltrierter Herd; in dessen Umgebung einige kleine Teleangiektasien. Ähnliche eben abgeheilte Herde an der medialen und lateralen Seite des linken Unterschenkels. Hier sind die Ränder bräunlich pigmentiert.

An den Händen und Beinen typische Skabies.

Innere Organe o. B. (Dr. Rosenthal, Medizin. Klinik).

Urin o. B.

Blutbild o. B. Blutplatten bleiben steril.

Pirquet und Intradermo für Tuberkulin, Trichophytin und Sporotrichin negativ.

Wassermann negativ.

Aus geschlossenen pustulösen Efflorescenzen an der linken Mamma: aerob und anaerob nur Staphylokokken.

Nach etwa anderthalb Monaten sind unter versehiedenen therapeutischen Maßnahmen (feuchten Verbänden, Ätzen, Kal.-Permanganatbädern), alle alten Herde zum Teil verheilt, zum Teil in Abheilung begriffen, der große Herd an der Brust fast verheilt. Dagegen hat sich am linken Oberschenkel aus einem ursprünglich längere Zeit klein bleibenden, infiltrierten Geschwür sehr rasch ein kleinhandtellergroßes Ulcus entwickelt. Es ist rund und zeigt einen abheilenden und einen fortschreitenden Rand. Dieser ist wulstig, derb, schmerzhaft, bläulichrot glänzend, seine Umgebung ödematös. Die Abheilung an der anderen Seite geht schr rasch vor sich, so daß eine Nierenform angedeutet wird. In der Mitte des Geschwürs sehr zahlreiche Granulationen (s. Abb.). Starke nächtliche Schmerzen in den Geschwüren. Am linken Unterschenkel, rechten Knie, sowie besonders in der Nähe des großen Herdes am rechten Oberschenkel entwickeln sich immer noch ne ue Herde (6. bis 8. Woche des Aufenthalts in der Klinik). Sie erscheinen als verhältnismäßig tief gelegene runde, schmerzhafte, flach gewölbte Infiltrate. In der Mitte dieser runden Papeln bilden sich weißliche Pusteln, die nach einigen Tagen spontan aufbrechen. Aus zwei frischen uneröffneten Herden: 1. Agarröhrchen steril, 2. Agarröhrchen: gramnegative, unbewegliche Stäbchen in größerer Menge. Nähere Artbestimmung s. u. An einem anderen Tage aus uneröffneten Efflorescenzen: Agar steril. Aus dem Rande offner Geschwüre mehrfach Bacillus pyocyaneus. Proteus.

Agglutination des Patientenserums mit dem aus einem Herd früher gezüchteten Bac. pyocyaneus bis $1: 160$ positiv.

Es besteht Neigung zu sekundären Pyodermien (Analabsceß, Furunkel, Schweißdrüsenabscesse der Achselhöhle). Behandlung mit Strubellscher Staphylokokkenvaccine.

Nach fast dreimonatigem. Aufenthalt in der Klinik zeigen die großen Herde kräftige Granulationen und rasche Überhäutung. Die Randzone der Geschwüre hat sich teilweise abgestoßen. In der Ungebung der gut abbeilenden alten Gc- 
schwüre treten noch immer ab und zu Knötchen auf, die am ersten Tage solid und rot sind, am zweiten Tage einen gelben Kopf zeigen. Verätzung mit Carbolsảure coupiert ihre Entwicklung.

Aus uneröfneter Papulopustel wird Pyocyaneus in Reinkultur gezüchtet, ebenso a us zwei nicht eröfneten Pusteln am rechten Unterschenkel, die nicht im Beginn verätzt worden and sich in 2 Tagen zu oberfläehlichen Abscessen entwickelt haber. Mit dem Eiter werden ein Meer-

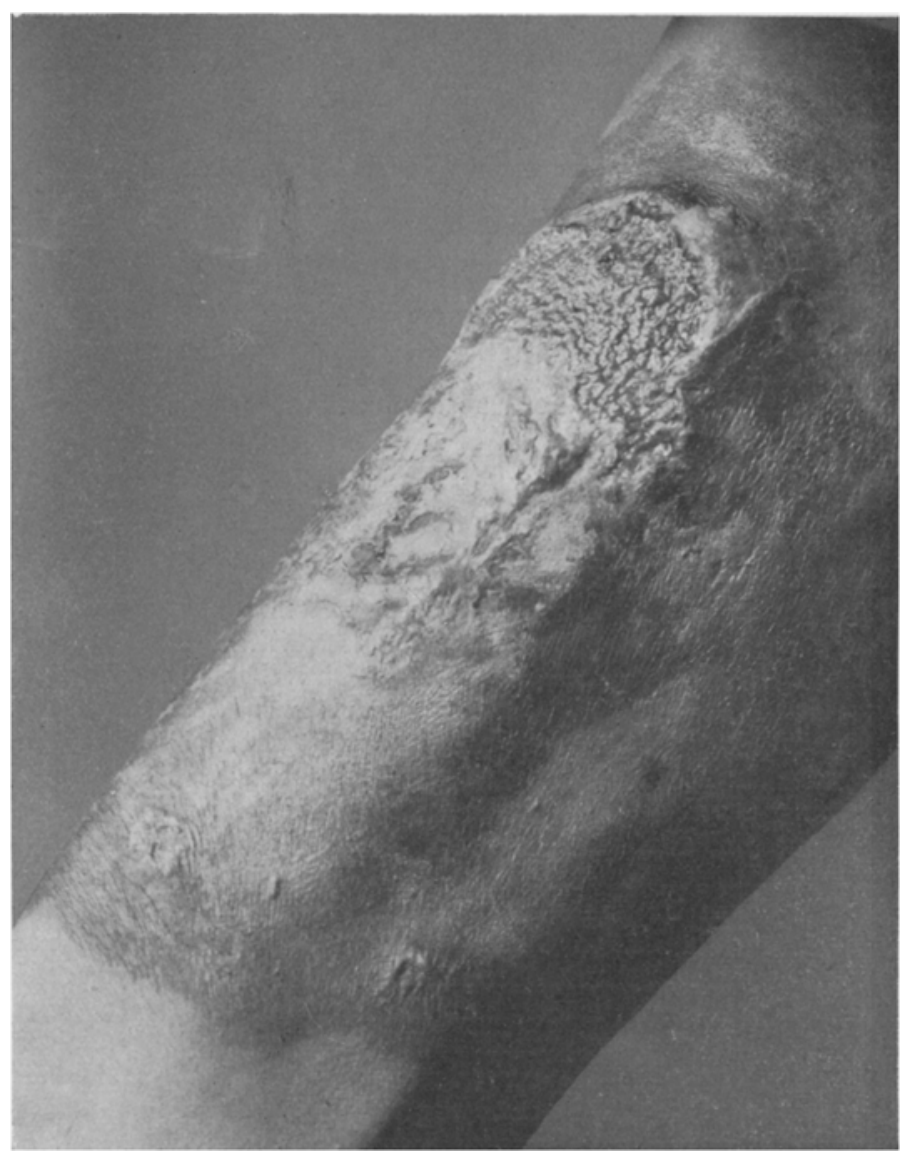

Voll ansgebildetes Geschwür am linken oberschenkel. Die dunkle Veriärbung in der Ungebung ruhrt yon der Behandlung mit Anthrarobintinktur her.

sehweinchen und eine Maus subcutan, eine weitere Maus intraperitoneal geimpit. Die Tiere bleiben gesund. Der gezüchtete Pyocyaneusstamm wird rom Patientenserum $1: 300+$ agglatiniort $(1: 200-)$.

Aus Gehörgängen, Sputum, Rachenschleim, Stubl, Urin ergaben Kulturen keinen Pyocyanexs.

Beginn einex Pyooyaneus-Autovaceinebehandlung: Intradermo 50 Willionen Keime. Am näohsten Tage fünffenniggroße leichte frischrote In- 
aus der Gruppe des Ecthyma gangraenosum (mit Pyocyanensbefund). 109

filtration, die nach 48 Stunden abklingt. Kochsalzkontrolle o. B. Erneute Impfung mit 50 Millionen hat den gleichen Erfolg.

Kontrollimpfung mit 50 Millionen Pyocyaneusvaccine bei 7 Frauen: Von diesen reagieren 6 (Ekzeme, Lues 2) mit geringer Rötung, die einen Tag andauert. Ein Fall mit einem seit 6 Jahren bestehenden, nun aber abgeheilten ulcerösen Tertiärsyphilid reagiert arn nächsten Tage mit flacher fünfpfenniggroßer Infiltration, die am folgenden Tage abklingt, und die fast der bei unserer Patientin gleicht.

Im Beginn der Vaccinebehandlung treten noch immer neue Efflorescenzen auf. Die Patientin bekommt von nun an jeden zweiten Tag erst um 25, dann um 50 Millionen steigende Dosen Pyocyaneusvaccine subcutan. Keine Reaktion. Nach einem Monat (4 monatige Behandlung) sind Einzeldosen von 500 Millionen erreicht. Geschwüre abgeheilt, Narben glatt, geringe Schrumpfung. Pyocyaneusvaccine + Lanolin anhydr. $\bar{a}$, auf frïher erkrankte und auf gesunde Haut aufgelegt, verursacht auf der ersteren ganz schwache Rötung. Agglutination bis $1: 640+$. Allgemeinbefinden gut. Entlassen.

Nach 6 Wochen schreibt Pat., daß es ihr gut gehe. Keine neuen Geschwüre. Das vom Arzt eingesandte Blut agglutiniert Bac. pyocyan. bis 1: 160 positiv.

\section{Histologische Untersủchung.}

Das aus einem Herd an der Brust am 4. VIII. 1920 exoidierte Stück zeigt ein Geschwür mit nicht sehr tief unterminiertem Rand. Der Grund des Geschwürs ist von roten Blutkörperchen und pyknotischem Eitermaterial bedeckt und weist nur sehr spärlich elastische Fasern auf. Nach unten zu wird die eitrige Infiltration weniger dicht und läßt Reste von kollagenem Bindegewebe erkennen, zwischen denen sich das pyknotisehe Material in Netzen und Strängen bis zur Schnittgrenze erstreckt. Nach der gesunden Seite zu wird das Infiltrat immer schwächer und ist noch ein Stück unter der Epidermis zu verfolgen. Dort, wo eitriges Material vorhanden ist, sind im Gegensatz zu den Resten des Bindegewebes die elastischen Fasern fast verschwunden. Im weiteren Umfange des Geschwürs ist das elastische Gewebe normal. Das Infiltrat besteht aus Leukocyten, nicht viel Plasmazellen und vereinzelten Eosinophilen.

Die Papillen sind stark hyperämisch. Vereinzelt haben Blutungen ins Stratum papillare und ins Epithel stattgefunden. An den Gefäßen, die nicht auffallend vermehrt sind, zeigt sich eine gewisse Endothelschwellung.

Das Epithel hört am Geschwürsrande ziemlich plötzlich auf. Es jst bis weit in die Umgebung hinein intra- und interepithelial ödematös mit dentlicher Bläschenbildung. In den Bläschen liegen Eiterkörperchen und ein feinkörniger Niederschlag. Das Ödem verliert sich nicht allmählich, sondern nach dem Schnittrande zu treten immer neue ödematöse Herde auf. Das Fpithel ist weithin im ganzen verdickt.

In der ganzen Breite des Schnittes bis zum Rande findet sich eine unbedeutende kleinzellige Infiltration und Vermehrung der Bindegewebselemente.

Parakeratose oder auffallende Vermehrung der Epithelmitosen auch im weiteren Umkreis sind nicht vorhanden.

\section{Biologische Untersuchungen.}

Die z. T. bereits in der Krankengeschichte kurz erwähnten bakteriologischen und serologischen Untersuchungen haben folgende Resultate ergeben: 
Bakteriologisches: Das Blut der Patientin wurde bei zweimaliger Untersuchung keimfrei befunden. Im Stuhl, Urin, Sputum, Rachenschleim sowie im Inhalt der Gehörgänge konnte Pyocyaneus nicht nachgewiesen werden.

Offene Geschwürsflächen wurden 3 mal untersucht; stets fand man reichlich Pyocyaneus, einmal vergesellschaftet mit Staphylokokken und Streptokokken, ein anderes Mal mit Proteus.

Siebenmal wurden geschlossene Efflorescenzen - stets nach sorgfältiger Desinfektion ihrer Oberfläche mit Alkohol und Äther - kulturell untersucht, meist zugleich aerob und anaerob unter Verwendung einer Reihe verschiedener Nährböden (Nährbouillon, Nähragar, Ascitesagar, Sabouraudschem Maltoseagar - auch bei Zimmertemperatur - Traubenzuckeragar in hoher Schicht).

In 2 Fällen blieben die Nährböden steril, ein mal w uchsen Staphylokokken und einmal gramnegative, unbewegliche dem Bact. pseudotubereulosis rodentium nahestehende, aber nicht mit ihm identische Stäbchen.

Diese Stäbchen hatten keine Geißeln, waren für weiße Mäuse nicht pathogen und zeigten kulturell auf den verschiedenen Nährböden folgende Eigenschaften: Bouillon diffus getrübt, Gelatineplatten nicht verflüssigt, auf ihnen ebenso wie auf Agarplatten die oberflächlichen Kolonien klein, grau-bräunlich, leicht schleimig, mit anfänglich glattem, später meist gekerbtem Rand, die tiefen Kolonien gelappt. Auf den verschiedenen Zuckernährböden weder Säure- noch Gasbildung, keine Indolbildung, Lackmusmolke schon nach einem Tag (37 ) gebläut.

Dreimal wurden aus Efflorescenzen, die bereits eitrig eingeschmolzen aber noch geschlossen waren, Pyocyaneusbacillen in Reinkultur gezüchtet. Der Eiter von 2 dieser Efflorescenzen wurde mikroskopisch untersucht, er enthielt polynucleäre Leukocyten und in einem Fall gramnegative schlanke Stäbchen in geringer Menge, die vereinzelt phagocytiert lagen, während in dem anderen Fall mikroskopisch keine Bakterien gefunden wurden. Mit dem ersten Eiter wurden 2 weiße Mäuse - die eine subcutan, die andere intraperitoneal -, sowie ein Meerschweinchen subcutan geimpft. An keinem der Tiere wurden Krankheitserscheinungen beobachtet.

Die 6 teils aus offenen Geschwüren teils aus geschlossenen Efflorescenzen gezüchteten Pyocyaneusstämme gaben im übrigen die t y pischen kulturellen und chemischen Reaktionen, nur bildete ein Sta $\mathrm{mm}$, der aus einer geschlossenen Efflorescenz in Reinkultur gewonnen war, kein Pyoc yanin, während er den grün fluorescierenden Farbstoff produzierte. Lag es schon im Hinblick auf die 5 anderen am gleichen Individuum gefundenen typischen Stämme an sich nahe, auch diesen Stamm als Pyocyaneus anzusprechen, der nur - im Körper oder beim Übergang auf den künstlichen Nährboden - die Fähigkeit zur Pyocyaninbildung eingebüßt hatte, so wurde diese Annahme be- 
wiesen durch. Untersuchungen über die Art seiner Begeißelung, durch die es gelang, ihn von der differentialdiagnostisch in Betracht kommenden Fluorescenzgruppe abzutrennen. Mittels der Pepplerschen Geißelfärbung ließ sich bei ihm ebenso wie bei den anderen Stämmen eine endständige Geißel darstellen, wie sie für Bact. pyocyaneum charakteristisch ist, während das Bact. fluorescens liquefaciens nach Burekhardt ${ }^{1}$ ) mehrere Geißeln besitzt. Außerdem besaß der Stamm die gleiche Meerschweinchenpathogenität wie die übrigen, indem er bei intraperitonealer Einverleibung von einer drittel Normalöse 24stündiger Agarkultur ein Tier innerhalb 24 Stunden unter dem Bilde einer serösen Peritonitis mit Blutungen in Pleura pulmonalis und Magenschleimhaut tötete. Dagegen gelang es nicht, ihm die Eigensehaft, Pyocyanin zu bilden, wieder anzuzüchten, weder durch kulturelle Verfahren (Züchtung in 2 proz. Peptonlösung mit und ohne Glycerinzusatz oder auf Glycerinagar) noch durch Tierpassagen (s. auch Phisalix und Charrin) ${ }^{2}$ ).

Entsprechend den alten Befunden von Gessard ${ }^{3}$ ) [s. auch Jakowski ${ }^{4}$ )] fanden auch wir, daß die Tierpassage eher eine Methode ist, die Pyocyaninbildung herabzusetzen als sie zu steigern.

Bəi einem der pyocyaninbildenden Patientenstämme haben wir, nachdem er beraits längere Zeit auf künstlichem Nährboden fortgezüchtet war, durch Plattengüsse das Verhältnis der pyocyaninbildenden zu den pyocyaninfreien Keimen [Baerthlein $\left.\left.{ }^{5}\right)\right]$ einerseits im Ausgangsmaterial, andererseits im Herzblut eines an intraperitonealer Impfung zugrunde gegangenen Meerschweinchens festgestellt.

Bəi der - unzureichenden - Beurteilung mit bloßem Auge fanden wir im Ausgangsmaterial 17 pyocyninhaltige und 28 pyocyaninfreie Kolonien, während im Material aus Herzblut das Verhältnis $2: 22$ betrug. Impften wir von beiden Platten Einzelkolonien auf Schrägagar ab und schüttelten die Schrägagarkulturen mit Chloroform aus, so erwiesen sich von je 9 Kolonien im Ausgangsmaterial noch 8 als pyocyaninhaltig, im Tiermaterial dagegen nur 3. Also bei beiden Beobachtungsreihen ergab sich, daß sich durch die Tierpassage das Verhältnis der Pyocyaninbildner zu den pyocyaninfreien Keimen zugunsten der letzteren verschoben hatte.

Immunitätsverhältnisse.

Die gezüchteten Stämme wurden mit Ausnahme der Staphylokokken - die Pyocyaneusstämme wiederholt - auf ihr Verhalten zu dem Patientenserum mittels Agglutination (makroskopische Methode, Beurteilung nach 2stündigem Aufenthalt bei $37^{\circ}$ ) untersucht. Die gramnegativen unbeweglichen Stäbchen wurden nicht agglutiniert, die verschiedenen Pyocyaneusstämme dagegen - vor Einsetzen der Behandlung mit Pyocyaneusvaccine (s. Krankengeschichte) - einmal bis zur Serumkonzentration $1: 160$, ein anderes Mal bis $1: 140$, der pyocyaninfreie Stamm dabei ebenso hoch wie die übrigen, während 3 normale 
Kontrollseren die Stämme in den Konzentrationen $1: 10$ bis $1: 160$ nicht agglutinierten.

Außerdem wurden 5 Sammlungsstämme des hiesigen Hygienischen Instituts dem Patientenserum gegenüber geprüft. 3 dieser Stämme wurden nicht agglutiniert, einer bis zur Konzentration $1: 40$, und der letzte bis zur gleichen Höhe wie die eigenen Stämme, d. h. bis zur Verdünnung $1: 140$, Befunde, die den Angaben der Literatur, daß innerhalb der Pyocyaneusgruppe verschiedene serologisch abzutrennende Arten bestehen, entsprechen.

Nach Abschluß der Vaccinebehandlung wurden erneut Agglutinationsreihen angelegt. Der Titer des Patientenserums war den eigenen Stämmen - auch dem pyocyaninfreien - sowie dem stark agglutinierten Sammlungsstamm gegenüber auf $1: 640$ gestiegen. 7 Wochen später war er gleichmäßig auf $1: 160$ zurückgegangen.

Die Tatsache, daß der pyocyaninfreie Stamm — vor der Vaccinebehandlung bis zur gleichen Höhe wie die übrigen Stämme der Patientin durch das eigene Serum agglutiniert wurde, spricht bis zu einem gewissen Grade gleichfalls für seine $\mathrm{Zu-}$ gehörigkeit zur Pyocyaneusgruppe. Man hat zwar vereinzelt gefunden, daß Pyocyaneusserum auch Bact. fluorescens agglutinierte [Eisenberg ${ }^{\natural}$ ), Pribam und Pula $\left.y^{7}\right)$ ], doch hat Trommsdorf ${ }^{8}$ ) an einem größeren Material zwischen beiden Gruppen serologisch keine Verwandtschaft feststellen können [s. auch Lang $\left.{ }^{9}\right)$ ]. Auch bei unseren Versuchen reagierte das Patientenserum gegenüber - allerdings nur - 3 Fluorescenzstämmen aus der Sammlung des Hygienischen Instituts in sämtlichen Konzentrationen negativ.

Ferner wurde das Patientenserum auf bakteriolytische Antikörper im Pfeifferschen Versuch gegenüber einem der eigenen Pyocyaneusstämme (Stamm mit Pyocyaninbildung aus geschlossener Efflorescenz) geprüft.

Zu dem Versuch wurden Meerschweinchen im Gewicht von 240-260 g verwandt. Durch Vorversuch wurde bei intraperitonealer Einverleibung als tödliche Grenzdosis $1 / 2 \mathrm{n}$-Öse 24 stündiger Agarkultur festgestellt.

Im Hauptversuch wurde den Tieren eine Normalöse gemischt mit je 0,4, 0,2, 0,1,0,05 und 0,025 $\mathrm{g}$ Patientenserum - durch Bouillon auf $1 \mathrm{~g}$ ergänzt verabfolgt. Als Kontrollen dienten 2 Normalseren in der Dosis von 0,4 und $0,2 \mathrm{~g}$, ferner ein Tier, das nur Bacillen ohne Serumbeimengung erhalten hatte.

Das Fortschreiten der Bakteriolyse wurde 4 Stunden hindurch beobachtet, indem in kurzen Zeitintervallen nach der üblichen Methode aus der Bauchhöhle mittels Glascapillaren Exsudat entnommen und im hängenden Tropfen untersucht wurde.

Bis zur Dosis von 0,05 herab konnten die bekannten Auflösungserscheinungen beobachtet werden: die Bakterien wurden rasch unbeweglich, quollen auf, wurden schattenhaft, bildeten auch Granula [s. dagegen $A$. Wasserma $n n^{10}$ ) und andererseits $\left.R_{4} a d z i e w s \mathrm{ki}^{11}\right)$ ] und waren nach 4 Stunden nur noch in ganz vereinzelten, unbeweglichen Exemplaren zu finden. Nur bei der Dosis von 0,05 hielten sie sich in etwas größerer Menge und blieben vereinzelt auch beweglich, Bei 0,025 war innerhalb der Beobachtungszeit keine erhebliche Veränderung an den Bakterien wahrzunehmen. Dagegen war bei den Kontrollseren nur die Konzentration von 0,4 wirksam - die Bakterien wurden unbeweglich, quollen auf und verminderten 
sich an Zahl - während die Menge von 0,2 g keine ins Gewicht fallende Reaktion hervorrief.

War somit eine spezifische Wirkung des Patientenserums im mikroskopischen Bilde festgestellt, so entsprach diesem Befunde doch nicht das Endergebnis. Zwar machten etwa 6 Stunden nach der Einspritzung die "Immuntiere" bis zur Injektion von 0,05 herab, sowie diejenigen ,Normaltiere", die $0,4 \mathrm{~g}$ Serum erhalten hatten, noch einen etwas muntereren Eindruck als die übrigen, doch waren am nächsten Morgen (18 Stunden nach der Injektion) alle Tiere tot. Bei allen wurde aus dem Herzblut Bact. pyocyaneum gezüchtet.

Wenn man nach diesem Endergebnis den Versuch auch nicht als gelungen bezeichnen kann, so hat sich doch bei der mikroskopischen Untersuchung im Patientenserum ein spezifischer Gehalt von bakteriolytischen Antikörpern feststellen lassen.

Endlich wurde, wie bereits oben angegeben (s. Krankengeschichte), auch in vivo mit Hilfe derintracutanen Reaktion festgestellt, daB die Patientin Antikörper gegen Pyocyaneus gebildet hatte. Als Material für diese diagnostischen - ebenso wie für die therapeutischen (s. Krankengeschichte) - Impfungen diente eine Mischvaccine, die aus den eigenen Stämmen, einem pyocyaninhaltigen und dem pyocyaninfreien zu gleichen Teilen, hergestellt war.

24stündige Agarkulturen in physiologischer Kochsalzlösung aufgeschwemmt, mit Glasperlen geschüttelt, durch Glaswolle filtriert, 1 Stunde auf $54^{\circ}$ erhitzt, auf Sterilität geprüft und mit $0,5 \%$ Phenol versetzt. Feststellung der Keimzahl nach der Wrightschen Methode (Blutausstriche).

\section{Epikrise.}

Bei einer anamnestisch unbelasteten Frau von 54 Jahren entwickelt sich, ohne daß anscheinend eine besonders schwächende Erkrankung vorangegangen wäre, eine im Verlauf chronische, in ihren einzelnen Efflorescenzen aber mehr oder weniger akut verlaufende ulceröse Hautaffektion, die nacheinander große Teile der Körperoberfläche ergreift. Die Lokalisation erscheint ganz regellos. Bald rezidivieren die Geschwüre in der gleichen Region, bald schießen in einer anderen neue Efflorescenzen auf. Rezidive in loco werden nicht beobachtet. Die Geschwüre entwickeln sich im späteren Verlauf der Erkrankung nur diesen konnten wir beobachten - aus kleinen Infiltraten, in deren Zentrum eine Pustel entsteht. Diese Pustel bricht nach einigen Tagen spontan auf. Die so entstandenen mit schmierigem grünen Eiter bedeckten Geschwüre schreiten mit bläulichrotem, wallartig erhabenem, z. T. unterminiertem, manchmal derb infiltriertem Rand nach einer oder allen Seiten sehr rasch fort und zerstören große Hautgebiete, ohne jedoch irgendwo tiefer als in die Subcutis zu gehen. Die Abheilung geht verhältnismäßig rasch vonstatten. Die Geschwüre verursachen z. T. spontan ziemlich erhebliche Schmerzen, doch ist eine ausgesprochene Berührungsempfindlichkeit nicht vorhanden. Die Narben sind 


\section{W. Frei und K. Wiener: Ein Fall von ulceröser Hauterkrankung}

verhältnismäßig weiß und zeigen weder Neigung zu Contracturen noch zu Keloidbildung.

Das Allgemeinbefinden ist im Verhältnis zur Sohwere des örtlichen Befundes ziemlich wenig gestört. Die Krankheit verläuft im wesentlichen fieberlos. Nach etwa einjährigem Kranksein und 4 monatiger klinischer Behandlung tritt Heilung ein. Die lokale Behandlung verschiedenster Art, besonders die Ätzung mit Carbolsäure, hatte wohl einen gewissen beschleunigenden Einfluß, doch hatte man niemals den Eindruck einer besonders energischen Einwirkming. Auch die Wirkung der Staphylokokkenvaccine war fraglich: a $m$ ehesten schien noch die Pyocyaneusvaccine Erfolg zo haben.

Bei Beginn der Behandlung wird als Nebenbefund eine Krätze festgestellt, später zeigen sich banale Pyodermien. Eine innere Medikation - insbesondere mit Brom - ist der Erkrankung nicht vorausgegangen.

Auf Grund der Anamnese und der klinischen, histologischen und serologischen Befunde können wir Lues, Tuberkulose, multiple neurotische Gangrän sowie andere ulceröse Hauterkrankungen, insbesondere Bromoderma, ausschließen und dürfen das Krankheitsbild ohne Zwang unter die in der Literatur als Ecthyma gangraenosum, Ecthyma térébrant oder multiple kachektische Hautgangrän beschriebenen Erkrankungen einreihen.

Unter den zuletzt genannten Namen sind in der letzten Hälfte des vorigen Jahrhunderts hänfig ulceröse Dermatosen beschrieben worden, die sich alle durch schnell wachsende, aus Papulopusteln entstehende Hautgeschwüre meist mit kraterartigem Rand und rotem Hof, auszeichnen. Es handelte sich in der Mehrzahl der Fälle um Kinder, die durch irgendwelche andere Krankheiten geschwächt waren, selten um Erwachsene, und nur ganz vereinzelt wird betont, daß die Exkrankung anscheinend Gesunde befiel. So wie sich klinisch mancherlei Verschiedenheiten der beschriebenen Fälle feststellen lassen, sind auch die verwertbaren bakteriologischen Ergebnisse nicht einheitlich, doch spielt unter den Befunden der Bac. pyocyaneus eine besondere Rolle (s. unten).

Auf die schon mehrfach und in den letzten Jahren von Takahashi' ${ }^{12}$ ) und Eite $\mathbf{l}^{13}$ ) besonders eingehend reproduzierte Literatur im einzelnen einzugehen, erscheint nicht mehr notwendig.

Verglichen mit den in der Literatur bekannten Fällen bietet unser Krankheitsbild mancherlei Eigentümlichkeiten, sowohl in klinischer wie in histologischer und bakteriologischer Beziehung. Zunächst ist das Alter der Patientin (54 Jahre) sehr ungewöhnlich, wenn auch vereinzelt [s. Lewandows $\mathrm{ky}^{14}$ ), Frä n kel ${ }^{15}$ ) u. a.] anscheinend hierhergehörende Erkrankungen in vorgerücktem Alter gefunden wurden. Ferner ist im Hinblick auf die Angaben der Literatur auffallend, daß es sich um eine sonst gesunde Frau handelt, die jedenfalls keine feststellbare als disponierend aufzufassende Erkrankung durchgemacht hat. Damit im Zusammenhang steht vielleicht auch der Ausgang in Heilung, der sonst äußerst selten ist. Merkwürdig ist weiter die 
aus der Gruppe des Ecthyma gangraenosum (mit Pyocyaneusbefund). 115

geringe Störung des Allgemeinbefindens sowie das Fehlen erheblicherer Temperatursteigerungen.

Die Efflorescenzen selbst stimmen in ihrer Entstehung aus Papeln, die sich über Pusteln zu schnell wachsenden Geschwüren entwickeln, im großen und ganzen mit den in der Literatur beschriebenen überein. Die Größe der Geschwüre scheint außer in einem von Walker und Cranston Law ${ }^{16}$ ) als Dermatitis gangraenosa infantum beschriebenen, auch sonst klinisch ähnlichen Fall, bei dem allerdings nur Staphylokokken gefunden wurden, nie die der Ulcera unseres Falles erreicht zu haben.

Die Zugehörigkeit unseres Falles zum Ecth y ma gangraenosum wird durch diese Eigentümlichkeiten wohl kaum in Frage gestellt. Dagegen muß erörtert werden, ob das von uns beschriebene Krankheitsbild mit den als Pyocyaneuserkrankungen bekannten Fällen von Ecthyma gangraenosum besondere Übereinstimmungen zeigt. Von den Hitschmann-Kreibichschen ${ }^{17}$ ) Fällen unterscheidet sich der unsrige durch das Fehlen eines besonders ausgesprochenen dunkelroten Hofes und durch die geringe Ausbildung der dort besonders betonten hämorrhagischen Entzündung. Während dort mehr eine trockene Nekrose beobachtet wurde, ließ sich bei unserer Kranken eine verflüssigende Form des Gewebstodes feststellen. Lew a ndows k y s Beschreibung der Läsionen stimmt am besten mit der unsrigen überein, doch war die Erkrankung viel leichter, da die Geschwüre auf den Unterschenkel beschränkt blieben. Ferner handelt es sich bei ihm auch nicht um eine gesunde, sondern eine tuberkulöse Patientin. Fränkel, der das bei weitem größte und einwandfreieste Material über Pyocyaneuserkrankungen zusammengetragen hat, schildert das durch den B. pyocyaneus hervorgerufene Ecthyma gangraenosum als eine Dermatose mit papulösen oder urticariellen schnell blasig werdenden Efflorescenzen, die rasch zu einer mehr trocknen Nekrose führen und dann scharfrandige Geschwüre mit rotem Hof bilden.

Die histologischen Befunde zeigen gegenüber denen von Hitschmann und Kreibich und von Fränkel erhebliche Unterschiede. Die ersterwähnten Autoren fanden durch den Verlust der Kernfärbbarkeit erkennbare mehr oder weniger tiefgehende Nekrose, der nur Reste von Drüsen und Follikeln widerstanden hatten. Es fehlte jedes entzündliche Infiltrat. Besonders auffallend war eine bei Methylenblaufärbung schon im Trockensystem als blaues Band sich abzeichnende Masse von Bakterien in den unteren Epidermisschichten. Auch die anderen nekrotischen Partien. waren diffus mit Bacillen durchsetzt, besonders stark die Gefäßwände. Fränkel sah in zahlreichen Fällen eine circumscripte Nekrose verschiedener Tiefe mit Ansammlung feinster gramnegativer Stäbchen in der äußeren Gefäß- 
wand. Auch bei ihm fehlt jede Leukocytenanhäufung, so daß er geradezu glaubt, daß der Bac. pyocyaneus negativ chemotaktisch auf die Leukocyten wirkt.

Ganz anders sind unsere Befunde: Fast keine nekrotischen Gewebsteile, keine Bakterien, aber ein auffallend starkes leukocytäres Infiltrat! Doch waren wiederholte Excisionen leider nicht möglich; die Untersuchung entstehender Efflorescenzen hätte vielleicht positive Bakterienbefunde gezeigt.

Es ergibt sich also aus dem Vergleich mit den beschriebenen sicheren Fällen von Pyocyaneuserkrankungen der Haut, daß diese in klinischer und histologischer Beziehung ein anderes Bild darboten mit Ausnahme des von Lewandowsky beschriebenen Falles, der allerdings nicht histologisch untersucht wurde. Die übereinstimmenden Punkte reichen jedenfalls nicht aus, um die Annahme, daß es sich bei unserem Fall um eine Pyocyaneuserkrankung handelt, zu beweisen.

Hierzu kommt noch, daß auch der bakteriologische Befund die ätiologische Bedeutung des Bact. pyocyaneum in unserem Fall nicht als völlig gesichert erscheinen läßt.

Die Tatsache, daß dieses Bacterium dreimal in geschlossenen Efflorescenzen in Reinkultur festgestellt worden war, und daß es im Organismus, wie der Ausfall der biologischen Reaktionen - Agglutination, Pfeifferscher Versuch, Allergieprüfung - bew eist, Antikörperbildung angeregt hatte, zwingt zwar dazu, ihm einen gewissen Anteil a $m$ Krankheitsprozeß einzuräumen. Doch schränkt seine Bedeutung schon der Umstand ein, daß zweimal in geschlossenen Efflorescenzen anscheinend gleicher Art andere Bakterienarten gleichfalls in Reinkultur -- gefunden worden waren, wenn auch der negative Ausfall der Agglutinationsprüfung bei einem der anderen Keime (den gramnegativen, unbeweglichen Stäbchen) vielleicht auf das Dominieren des Bact. pyocyaneum hinweist. Ferner muß man noch berücksichtigen. daß zweimal die kulturelle Untersuchung geschlossener Efflorescenzen überhaupt negativ verlaufen war. Wenn man daraus den Schluß ziehen wollte, daß in diesen. beiden Fällen die Efflorescenzen keine Keime enthalten hätten, so würde das dafür sprechen, daß sich in den übrigen Fällen die Bakterien nur sekundär im erkrankten Gewebe angesiedelt hatten; wenn man dagegen damit rechṇet, daß auch. hier Mikroorganismen vorhanden waren, und nur ihre Kultivierung nicht gelungen war, dann liegt es näher, den Keimen - und unter diesen vielleicht vorherrschend dem Bact. pyocyaneum - einen Anteil an der Genese der Efflorescenzen einzuräumen.

Zugunsten der letzteren Anschauung könnte man das Ergebnis der Behandlung mit Pyocyaneusvaccine (s. Krankengeschichte) 
aus der Gruppe des Ecthyma gangraenosum (mit Pyocyaneusbefund).

anführen. Während sich vor Beginn der Impfungen und etwa eine Woche über die Erstimpfung hinaus noch neue Herde gebildet hatten, hörte von dieser Zeit an die Neubildung von Efflorescenzen auf, und die vorhandenen gingen in Heilung über. Doch muß man wiederum einschränkend in Betracht ziehen, daß sich die Patientin bereits vor dem Einsetzen, der Vaccinebehandlung entschieden auf dem Wege der Besserung befand.

Unabhängig von der Frage, ob die Keime an der Entstehung der Efflorescenzen beteiligt waren oder sich nur sekundär angesiedelt hatten, kann man wohl annehmen, daß sie auf ektogenem und nicht auf hämatogenem Wege eingewandert waren. Dafür sprechen der relativ leichte Verlauf der Erkrankung - trotz der Schwere des lokalen Prozesses - , die - zweimal festgestellte - Sterilität des Blutes, die Mannigfaltigkeit der in den Efflorescenzen aufgefundenen Bakterienflora sowie bis zu einem gewissen Grade der Umstand, daß der hauptbeteiligte Keim, das Bact. pyocyaneum, an seinen anderen Prädilektionsstellen im Körper (Ohr, Lunge, Harnwege, Darm) bei der bakteriologischen Untersuchung der Ex- und Sekrete nicht festzustellen gewesen war. Wieweit bei der Inokulation der Keime Kratzen infolge der Scabies eine Rolle gespielt hat, ist nicht mit Sicherheit zu sagen.

Ein Versuch auf tierexperimentellem Wege die Ursache für das $A b-$ weichen des von uns beobachteten Krankheitsbildes vom Typus der Pyocyaneuserkrankungen der Haut zu finden, führte zu keinem positiv verwertbarem Resultat.

Einmal nahmen wir die Tatsache, daß Hitschmann und Kreibich sowie Fränkel bei ihren histologischen Untersuchungen große Bacillenmengen in den Efflorescenzen gefunden hatten, während uns der histologische Bacillennachweis überhaupt nicht gelang, zum Ausgangspunkt und infizierten Meerschweinchen intracutan teils mit relativ großen ( $1 / 10$ Normalöse 24 stündiger Agarkultur), teils mit kleinen $(1 / 10000$ Normalöse) Bakteriendosen.

Andererseits gingen wir von der Überlegung aus, daß in unserem Falle die Erkrankung nicht wie gewöhnlich einen stark geschwächten Organismus betroffen hatte, und daß ferner infolge der langen Dauer der Erkrankung in unserem Falle die Antikörperbildung möglicherweise erheblicher gewesen war als sonst, und verglichen infolgedessen den Verlauf dermaler Pyocyaneusinfektionen bei geschwächten (tuberkulösen oder hungergeschädigten) Tieren mit dem Verlauf bei normalen und bei immunisierten Tieren (die durch 3 malige intraperitoneale Impfung mit abgetöteten Pyocyaneusbacillen vorbehandelt waren).

Die Infektion wurde nach Rasieren der Hautstellen teils durch Einbringen. der Bakterien in vorpräparierte Hauttaschen, teils durch intradermale Injektion gesetzt.

Die Impfungen mit kleinen Bakterienmengen zeitigten in keinem Fall sichtbare Krankheitserscheinungen; unter der Einwirkung größerer Mengen bildeten sich überall etwa $1-4 \mathrm{qcm}$ große, derbe, rote. Infiltrate mit eingesunkenem hämorrhagischem, später mit einer Kruste bedecktem Zentrum, die ohne wesentliche Geschwürsbildung abheilten. Ins Gewicht fallende 
118 W. Frei und K. Wiener: Ein Fall von ulceröser Hauterkrankung usw.

Unterschiede zwischen den verschiedenen Gruppen wurden nicht beobachtet.

\section{Zusammenfassung.}

Bei einer anscheinend gesunden, nicht kachektischen, älteren Frau treten an den verschiedensten Körperstellen ein Jahr hindurch papulöse schnell pustulös werdende Infiltrate auf, die geschwürig zerfallen. Die schmierig-eitrig-grünlich belegten Geschwüre nehmen durch regelmäßiges oder unregelmäßiges peripheres Fortschreiten mit infiltriertem und unterminiertem Rand große Dimensionen an, haben aber eine ausgesprochene spontane Heilungstendenz.

Therapeutisch schien am ehesten Pyocyaneusvaccine zu wirken. Trotz dieser Beobachtung und des Befundes von Pyocyaneus in einzelnen uneröffneten Efflorescenzen sowie der positiven serologischen und Allergieversuche ist es uns nicht möglich, die Zugehörigkeit unseres Falles zu den primären Pyocyaneuserkrankungen der Haut mit Bestim m theit $\mathrm{z} u$ beha u pten. Immerbin ist die ätiologische Beteiligung des Bact. pyocyaneum an dem Krankheitsprozeß in einem gewissen Maße wahrscheinlich gemacht worden.

Es muß der Untersuchung analoger Fälle vorbehalten bleiben, darüber zu entscheiden, ob das beschriebene Krankheitsbild den Pyocyaneuserkrankungen der Haut zuzurechnen ist, und ob es unter dieser schon von Fränkel als nicht einheitlich gekennzeichneten Gruppe - vielleicht mit dem Fall Lewandowskys zusammen - einen besonderen Typus darstellt.

\section{Literatur.}

1) Burckhardt, Centralbl. f. Bakt. Orig. 79, 321. 1917. - 2) Phisalix und Charrin, Rof. ebenda 14, 429. 1893. - ${ }^{3}$ ) Gessard, Ann. de l'Inst. Pasteur 5, 65. 1891. - $\left.{ }^{4}\right)$ Jakows ki, Zeitschr, f. Hyg. 15, 474, 1893. - $\left.{ }^{5}\right)$ Baerthlein, Centralbl. f. Bakt. Ref. 54, 178*. 1912. (Tagungsbericht.) - 6) Eisenberg, Ebenda Orig. 34, 739. 1903. - ${ }^{7}$ ) Priba m und Pula y, Ebenda 76, 321. 1915. 8) Trommsdorf, Ebenda 78, 493. 1916. - ${ }^{9}$ ) Lang, Ref. ebenda 62, 213. 1914. - ${ }^{10}$ ) A. Wassermann, Zeitschr. f. Hyg. 2z, 263. 1896. — ${ }^{11}$ ) Radziewski, Ebenda 37, 1. 1901. - 12) Takahashi, Diese Zeitschr. 120, 739. 1914; Dermatol. Zeitschr. 21, 702. 1914. - ${ }^{13}$ ) Eitel, Inaug.-Diss. Berlin 1915. - 14) Lewandowsky, Münch. med. Wochenschr. 1907, S. 2275. — 15) Fränkel, Virchows Archiv 183, 405. 1906; Zeitschr. f. Hyg. 72, 486. 1912; 84, 369. 1917. - ${ }^{16}$ ) Walker und Cranston Low, Ikonographia dermatol. 4, 141. 1909. — ${ }^{17}$ ) Hitschmann und Kreibich, Wiener klin. Wochenschr. 1897, S. 1093; diese Zeitschr. 50, 81, 1899 . 